\title{
Parental presence at the bedside of critically ill children in the pediatric intensive care unit: A scoping review
}

\author{
Lauren Miller ${ }^{1} \cdot$ Monique Richard $^{2} \cdot$ Kristina Krmpotic $^{2,3}$ (C) Anne Kennedy ${ }^{4}$ Jamie Seabrook $^{5,6,7}$ ([) \\ Corey Slumkoski ${ }^{8} \cdot$ Martha Walls $^{8} \cdot$ Jennifer Foster ${ }^{2,3,7}$ (D)
}

Received: 30 June 2021 / Revised: 14 September 2021 / Accepted: 30 September 2021 / Published online: 9 October 2021

(c) The Author(s), under exclusive licence to Springer-Verlag GmbH Germany, part of Springer Nature 2021

\begin{abstract}
Parental presence at the bedside (PPB) of critically ill children in the pediatric intensive care unit (PICU) is necessary for operationalizing family-centred care. Previous evidence syntheses emphasize parent-healthcare provider interactions at rounds and resuscitation; our focus is the parent-child dyad. Prior to embarking on further study, we performed a scoping review to determine the breadth and scope of the literature addressing PPB of critically ill children in the PICU. We searched five online databases (MEDLINE, EMBASE, CINAHL, Cochrane Library, and PSYCHINFO) and the grey literature to identify English and French reports from January 1960 to June 2020 addressing physical parental presence with children (birth to 18 years) in intensive care units, without limitation by methodology. Screening, reference selection, and data extraction were performed by two independent reviewers. Data were extracted into a researcher-designed tool. We identified 204 publications (81 quantitative, 68 qualitative, 22 mixed methods, and 9 descriptive case or practice change studies, and a further 24 non-study reports). PPB was directly assessed in 78 (38\%) reports, and was the primary objective in 64 (31\%). Amount or quality of presence was addressed by 114 reports, barriers and enablers by 152 sources, and impacts and outcomes by 134 sources. While only 6 reports were published in the first two decades of our search (1960-1980), 17 reports were published in 2019 alone.

Conclusions: A relatively large body of literature exists addressing PPB of critically ill children. Separate systematic evidence syntheses to assess each element of PPB are warranted.

Scoping review protocol registration: Open science framework, protocol nx6v3, registered 9-September-2019.

\section{What is Known:}

- Parental presence at the bedside of critically ill children must be enabled to facilitate family centeredness in care.

- Systematic evidence syntheses have focused on parental presence at rounds or resuscitation, rather than with the child throughout the intensive care journey.

What is New:

- Many reports $(n=204)$ address parental presence at the bedside in the pediatric intensive care unit, though most do as incidental findings

- Identifies studies addressing key elements of parental presence in the PICU including barriers and enablers to, amount and quality of, and

impact and outcomes of parental presence, and demonstrates trends over time and geography.
\end{abstract}

Keywords Parent $\cdot$ Child $\cdot$ Visitation $\cdot$ Family presence $\cdot$ Pediatric intensive care unit $\cdot$ Scoping

Abbreviations
$\begin{array}{llll}\text { COVID-19 } & \text { coronavirus disease } 2019 & \text { PPB } & \text { Parental Presence at the Bedside } \\ \text { NICU } & \text { Neonatal intensive care unit } & \text { PICU } & \text { Pediatric Intensive Care Unit }\end{array}$

Communicated by Piet Leroy

Jennifer Foster

jennifer.foster@iwk.nshealth.ca

Extended author information available on the last page of the article

\section{Introduction}

For critically ill children admitted to the pediatric intensive care unit (PICU), parental presence at the bedside (PPB) is a source of comfort [1] and a recognized human right [2]. 
Historically, visiting hours and lack of privacy limited PPB [3]. In today's evolving healthcare climate, a more familycentered approach has addressed some of these issues through pediatric critical care teams encouraging parental presence and involvement in care [4, 5]. As has become evident in the wake of the coronavirus disease 2019 (COVID19) pandemic, unlimited parent and family presence may face significant barriers and is still seen as an optional element of care $[6,7]$. Before designing research on PPB, an understanding of the scope of existing literature on the topic is needed.

Preliminary searches of PROSPERO, MEDLINE, the Cochrane Database of Systematic Reviews, CINAHL, and JBI Evidence Synthesis prior to protocol development (February 11,2019$)$ and to manuscript preparation (June 11, 2021) did not identify any systematic or scoping reviews on the topic. A 2018 integrative review examined whether research on parental perspectives of family-centered care demonstrated implementation of its 4 core concepts, which include participation in care but not presence at the bedside specifically [8]. A systematic review of hospital visitation policies explored reported impacts but was not PICU-specific and examined policy rather than actual presence [9]. A systematic review of PICU parental presence during resuscitation and invasive procedures identified that being present improved coping and satisfaction with care [10] but did not address presence through the entirety of the admission.

We define PPB as physical parental presence in the PICU room with their critically ill child. The presence may be active, in which the parent is engaged in provision of care, or passive, in which the parent is present though not involved in providing comfort or care. Unlike research focusing on parental presence on rounds, which emphasizes the parenthealthcare team interactions, our research question focuses on the parent-child dyad. Based on our literature review and clinical experience and expertise as PICU clinicians, our study team conceptualizes that studies addressing PPB may consider the amount and nature of time spent together in the PICU, barriers and enablers to parent presence with their critically ill child, and the impact of this interaction on health-related outcomes (Fig. 1).

The amount and nature of time that parents spend with their critically ill child is unknown, as is the quantity and type of literature addressing the topic. There may be multiple barriers or enablers that impact PPB, not least of which are restrictions triggered by the COVID-19 pandemic. [11, 12]. One can conceptualize that hospital and PICU policy and practice [13], elements of the child's illness [3, 14], PICU environment [13, 15], external factors such as siblings [16], and parents' intrinsic motivations and capabilities [17] may all act as barriers or enablers to presence, though the extent of the literature addressing the topic is unknown. Finally, preliminary evidence in adults demonstrated associations between unrestricted family presence policy and improved bio-[18], psycho-[19] social [20] outcomes. While it seems intuitive that PPB would benefit children, even if potentially stressful or distressing for parents, the evidence has not been systematically explored. There is a need to develop an understanding of family and parental presence in the PICU, and to identify what elements have been investigated. Prior to undertaking such an assessment, a scoping review is needed to systematically map the existing literature. The primary objective of this review was to detail the breadth and depth of the current body of literature addressing PPB of critically ill children in PICU, including the number and types of studies addressing each of the concepts outlined in the researcher-proposed PPB framework (Fig. 1). We secondarily sought to understand the breadth of literature that directly investigates PPB and to identify the works for which an aspect of PPB was the primary objective. This overview will be used to determine the need for and feasibility of future investigations, including systematic reviews, in the area.
Fig. 1 Proposed framework for elements of Parental Presence at the Bedside (PPB)

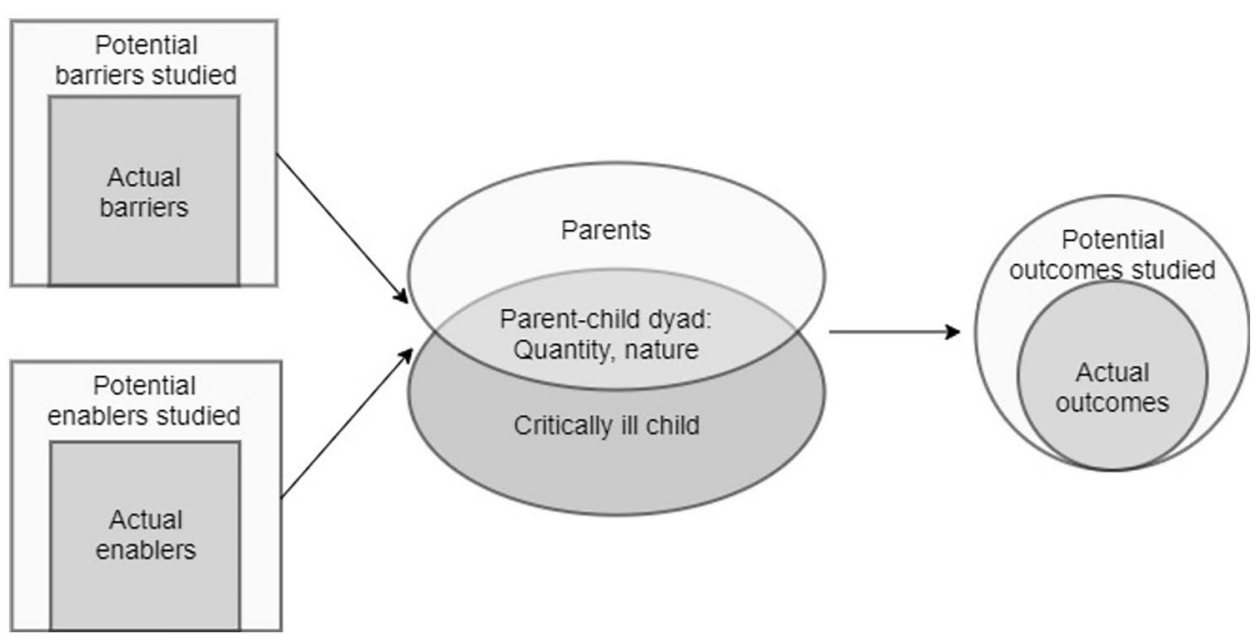




\section{Methods}

\section{Review question}

What is the scope and nature of literature addressing parental presence at the bedside of critically ill children in the PICU?

\section{Study design}

This scoping review was conducted using the methodology described by Arksey and O'Malley [21] and the enhancements proposed by Levac and colleagues [22]. The protocol was registered prospectively with the Open Science Framework on 9 September 2019 (osf.io/nx6v3). The Preferred Reporting Items for Systematic reviews and Meta-Analyses extension for Scoping Reviews (PRISMA-ScR) checklist was used in designing and reporting the study and is available as Supplementary File 1.

\section{Eligibility criteria}

The search started at 1960, which marks the earliest references to pediatric intensive or critical care units in the published literature, and ended in 2020 (Database searches June 20, 2020, website searches July 15, 2020), enabling assessment of trends over time. The search was limited to records published in English or French, the working languages of the study team.

Population: Studies were eligible for inclusion if they described parents of critically ill children (ages birth to 18 years [23]) who were admitted to a PICU. We included studies that defined parents in any study-defined manner. Studies that considered visitors generally without specific reference to parents were not included.

Context: As the context of interest we included all PICUs, including trauma, neuro-critical care, burn, cardiac, medical, and surgical units, or critical care units. We included mixed NICU-PICU and adult intensive care unit-PICU studies where pediatric patients and their families were clearly defined and distinguishable. We included step-up/step-down units where the described scope included acute therapy or monitoring that would normally be delivered in a PICU (e.g., non-invasive ventilation, continuous salbutamol [23]). Studies limited to descriptions of parents or families of patients in NICUs were not included as the context and culture of NICUs differ from those of PICUs [24].

Concept: Included studies specifically addressed the concept of PPB, defined as active or passive physical presence of the parent(s) in the PICU with the child. Studies addressing family-centered care (FCC) generally or familycentric models of care were only included if they contained a description of parental presence, or of the impact or outcome of parental presence with respect to the care model. For the purposes of this review, barriers and enablers are conceptualized within a Capabilities, Opportunities, Motivations behavior change framework [25] as factors that may influence the ability, drive, and opportunities for the parents to be present at the bedside. The quantity and nature of the time spent at the bedside is conceptualized as any measure or count of time or events of parents at the bedside, or descriptions of the nature, quality, or type of presence at the bedside including active participation in care activities (e.g., bathing, soothing) and passive presence (e.g., sitting at the bedside). Patient care rounding was only considered when the study detailed rounds at the bedside to enable realization of the child-parent dyad. Outcomes are broadly considered as any impact on physiologic, psychologic, and/or psychosocial health of patient, family members, or healthcare providers or impact on nature and delivery of care.

Study types: Reports were not limited by methodology. In addition, text and opinion papers were considered for inclusion as we anticipated that they may address perceived barriers and enablers, nature, and outcomes of PPB. We included published conference abstracts but excluded the abstract when a subsequent, corresponding peer-reviewed publication could be identified that contained all the information from the abstract. Systematic evidence syntheses and literature reviews that reported on aspects of PPB were not included; however, their citation lists were reviewed for primary studies that may have met eligibility criteria.

\section{Information sources and search strategy}

Search strategies were drafted by LM in collaboration with an experienced research librarian and included the following concepts: Pediatric; Intensive care unit; Parents. Five articles known to fit inclusion criteria were identified [11-13, 26, 27], and their keywords were used to assist in the design of the initial search strategy. The final search strategy for MEDLINE can be found in Supplementary File 2 and was adapted for EMBASE, CINAHL, Cochrane Library, and PSYCHINFO. Bibliographic databases were searched from January 1960 to June 20, 2020. We scoped the grey literature (July 15, 2020) for relevant manuscripts and abstracts by searching OpenGrey.eu, New York Academy of Medicine's Grey Literature Report, the first 100 hits in Google Scholar, and the websites of the Society for Critical Care Medicine, American Association of Critical Care Nurses, American Academy of Pediatrics, European Society of Pediatric and Neonatal Intensive Care, European Federation of Critical Care Nurses, Canadian Critical Care Society, Critical Care Forum Canada Academy, Canadian Association of Critical Care Nursing associations, Canadian Pediatric Society, Planetree International, and Institute for Patient and Family 
Centered Care. The final search results were exported into Covidence software (Covidence, Melbourne, Australia), and duplicates were removed. The electronic database search was supplemented with backward reference chaining of all included reports.

\section{Study selection process}

Two independent reviewers screened citation titles and abstracts for potential inclusion, erring on inclusion for full text review. Pilot screening, whereby records were reviewed 10 at a time with follow-up team review, proceeded until reviewers agreed $>90 \%$ of the time. Inter-rater discrepancies were resolved by discussion or by a third, independent reviewer if consensus could not be attained. The inclusion/ exclusion criteria were revised during pilot screening to exclude studies that addressed PPB in an exclusively NICU setting; the significant differences in NICU culture and practices [24] made the context outside the scope of the study objectives for this PICU-focused synthesis.

The full text reports for records retained after title and abstract screening were obtained and imported into Covidence software. Articles were then assessed for inclusion in the final review following the same process as during title and abstract screening. Reason for exclusion was recorded for all reports that were not included at this stage.

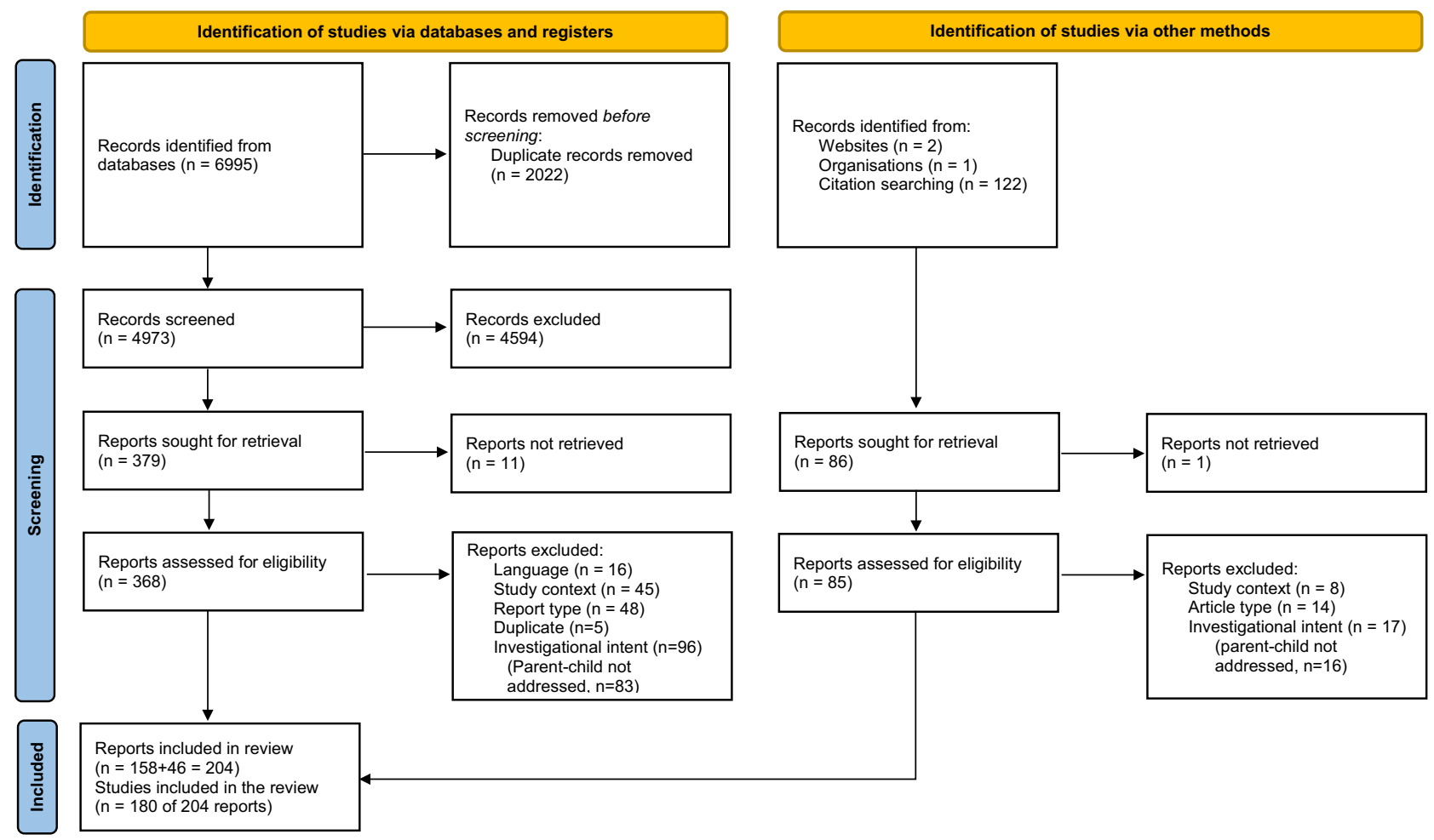

\section{Data extraction}

A data charting form was developed a priori, pilot tested with 5 articles, and revised to optimize extraction efficiency. The data extraction form was modified to remain consistent with the study question and objectives when the objectives changed for feasibility. Given the high volume of literature, we removed the objectives of extracting and summarizing all elements of PPB that were studied and the results of those studies. Two members of the review team independently extracted and charted the data, resolving discrepancies by discussion or, if consensus could not be attained, by a third independent reviewer.

We extracted data on article characteristics (author, year of publication, country of origin), study characteristics (study methodology [e.g. qualitative, quantitative, mixed methods, other], methods, primary objective, number of study sites, sample size, population), aspect of PPB addressed (barriers/enablers, amount/quality, outcomes) and whether it was directly assessed or indirectly addressed, and whether PPB was the primary objective or outcome. We defined direct assessment as one in which PPB was assessed through either the primary or secondary outcomes, or intentional assessment and reporting of an element of PPB through structured or semi-structured methods as part of the study tools. We defined a study as having indirectly

Fig. 2 PRISMA 2020 flow diagram for identification, screening, assessment and inclusion of reports 
addressed PPB when elements of the proposed PPB framework were reported as a finding without being the purpose of the article, specifically sought, or when elements were part of a questionnaire but the results as it pertains to PPB were not specifically reported.

Findings were summarized descriptively using counts and percentages, with studies separated according to whether they addressed PPB directly or indirectly, the elements of PPB that were addressed, and the type of study or report.

\section{Results}

We identified 6995 records through database searches. After de-duplication and screening $(n=4973)$ we reviewed the full text of 379 reports. Most reports $(n=96)$ were excluded for investigational intent inconsistent with the review question; in 83 of these, the parent-child dyad was not addressed. We identified 158 reports for inclusion. A further 125 records were identified for assessment through screening the citations of all included reports and examination of websites, with 46 assessed as meeting inclusion and no exclusion criteria. A total of 204 reports that studied or reported on PPB in the PICU were included in the scoping review (Fig. 2).

Table 1 outlines the study type, whether PPB was addressed directly or indirectly, and whether each of the elements in the PPB framework (Fig. 1) were addressed directly or indirectly. The references for reports associated with each cell in Table 1 are supplied as Supplementary File 3 and the data charted to address the study objectives are supplied as Supplementary File 4 . The reports included 180 studies: 68 qualitative, 81 quantitative, and 22 mixed methods studies, and 9 descriptive studies of cases or practices changes. We also identified 12 expert opinions, commentaries, or editorial pieces, 4 practice points, 5 reflective pieces, and 3 clinical practice guidelines or position statements. There were $8 \mathrm{PhD}$ dissertations and 19 reports in abstract form only.

Sixty-four (31\%) reports addressed parental presence as a primary objective. Most reports (62\%) addressed PPB indirectly, with only $38 \%$ assessing one or more elements of PPB intentionally.

We identified that: 152 (76\%) reports addressed barriers or enablers to PPB, including factors related to parent and child characteristics, healthcare provider attitudes, policy and PICU practice, and program implementation; $114(56 \%)$ addressed the amount or quality of time spent at the bedside; and $134(66 \%)$ addressed outcomes of $\mathrm{PPB}$, including outcomes related to health and mental health of the patient and parents, medical practices and patient safety, and healthcare provider experiences and attitudes. Categorically, 66 (32\%) addressed a single area, $80(39 \%)$ addressed 2 areas, and $58(28 \%)$ addressed all three areas. However, in readdressing only those elements assessed directly, 2 or 3 elements were simultaneously addressed in $60 \%$ of the studies (Fig. 3).

Literature addressing PPB was infrequently published before 2005, after which time it has steadily increased (Fig. 4). Publications originated from 29 different countries (Fig. 5), with the majority from the United States $(\mathrm{n}=97,48 \%)$, Canada $(\mathrm{n}=26,13 \%)$, and the United Kingdom $(n=18,9 \%)$.

\section{Discussion}

This is the first scoping or systematic review to determine the scope and type of literature addressing PPB and was strengthened by following rigorous methodology and the PRISMA-ScR guidance. This scoping review identified a relatively large body of literature addressing PPB of critically ill children, and a smaller but important body of literature assessing PPB either in the objectives or as part of a study tool. The scope of the literature identified was so broad and varied that, to ensure review feasibility, it necessitated eliminating the intended objectives to

Table 1 Description of included studies by study type, and whether parental presence at the bedside (PPB) was assessed directly or indirectly. Direct or indirect assessment is indicated overall for each study type, and for each element of PPB (barriers and enablers, amount and type, or outcomes)

\begin{tabular}{lll}
\hline & Direct & Indirect \\
\hline & $n=78(38 \%)$ & $n=126(62 \%)$ \\
Qualitative (n=68) & $\mathbf{8}$ & $\mathbf{6 0}$ \\
Barriers/enablers & 4 & 55 \\
Amount/quality & 5 & 35 \\
Outcomes & 3 & 47 \\
Quantitative (n=81) & $\mathbf{5 1}$ & $\mathbf{3 0}$ \\
Barriers/enablers & 18 & 27 \\
Amount/quality & 22 & 20 \\
Outcomes & 32 & 23 \\
Mixed Methods (n=22) & $\mathbf{9}$ & $\mathbf{1 3}$ \\
Barriers/enablers & 3 & 13 \\
Amount/quality & 4 & 8 \\
Outcomes & 6 & 8 \\
Descriptive cases/ practice & $\mathbf{4}$ & $\mathbf{5}$ \\
change (n=9) & & \\
Barriers/enablers & 4 & 5 \\
Amount/quality & 3 & 1 \\
Outcomes & 1 & 5 \\
Other (n= 24) & $\mathbf{6}$ & $\mathbf{1 8}$ \\
Barriers/enablers & 5 & 19 \\
Amount/quality & 2 & 14 \\
Outcomes & 2 & \\
\hline & &
\end{tabular}


Fig. 3 Reports addressing aspects of parental presence at the bedside (Fig. 1), considering only those reports that assessed an element directly through primary or secondary outcome, or as a direct measure in a study tool

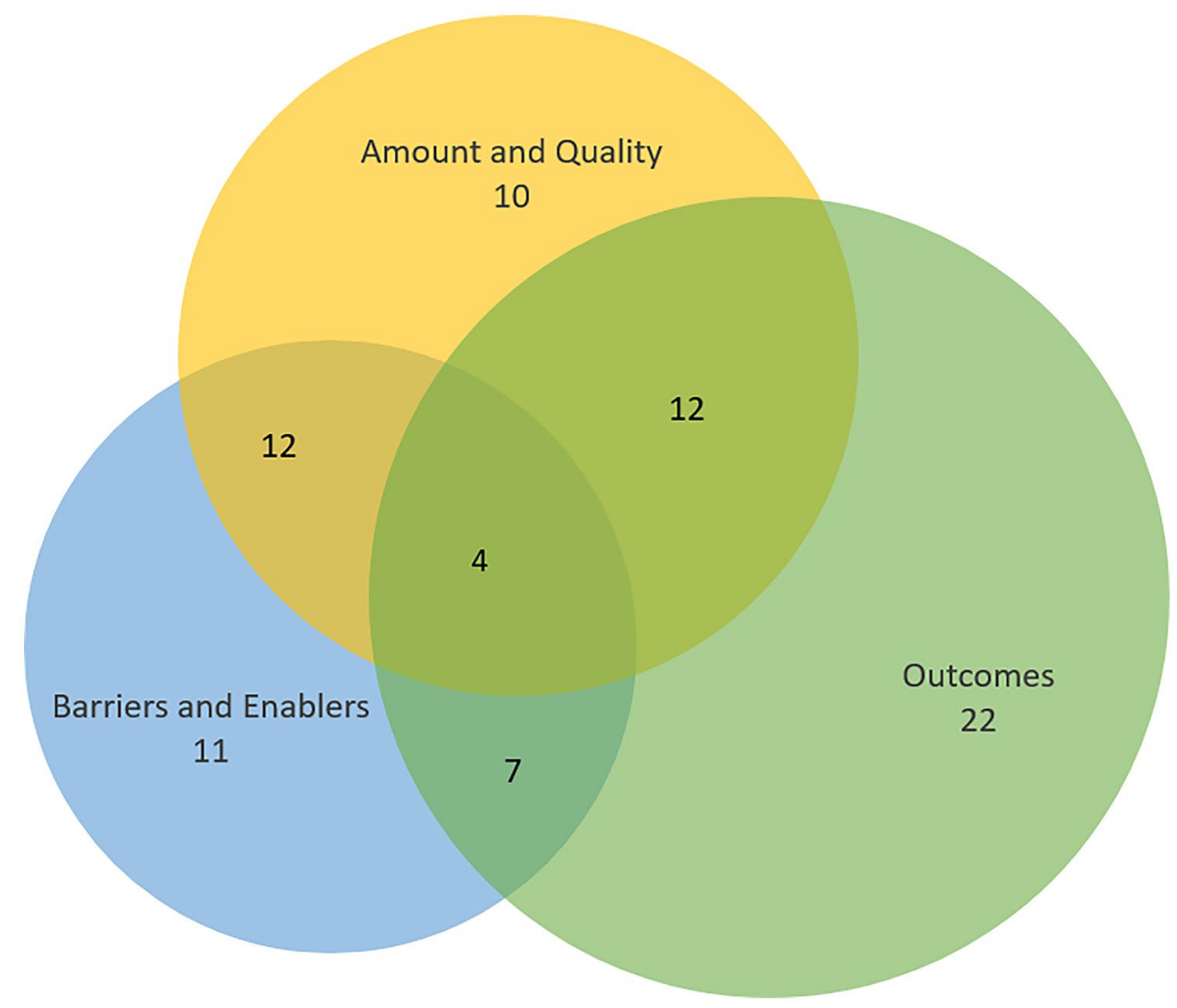

chart the exact elements studied and the results of those investigations. Acknowledging this limitation, we have identified a need to follow this scoping review with systematic evidence syntheses in each of the three elements of the researcher-proposed PPB framework: barriers and enablers; amount and nature; and outcomes.

Most literature addressing PPB does so only incidentally. It is notable that quantitative research was more likely to directly assess PPB_-particularly outcomes-and that qualitative studies were more likely to incidentally address the topic. As most included qualitative studies involved open-ended interview questions, we surmise that parents are raising PPB for discussion more frequently than they are being explicitly asked about it. This topic - which is clearly important to families - warrants further direct assessment. Although further systematic evidence syntheses should include only those studies that address PPB as a primary or secondary outcome measure, future researchers may be assisted and inspired by references addressing the topic incidentally.

This review identified a clear increase in the PPB-related literature over the last 20 years, which parallels the increase in general publication volume; MEDLINE searches for "family centered care" or "pediatric intensive care" demonstrate similar increases in the literature volume over the same years. Although we only identified 8 reports in the first half of 2020, we anticipate that the sweeping restrictions to family presence implemented during the COVID-19 pandemic [13] will lead to a significant increase in reports addressing the issue. This may warrant a specific review to highlight COVID-19 related PPB literature in the coming years.

Our study also highlights the narrow lens through which most of the research addressing PPB has occurred, with Westernized nations accounting for the vast majority of publications. This may be somewhat expected by our review's language limitations (French and English). Also, the Global South is still developing its abilities to provide pediatric critical care, [28] and may lack the governmental support for research on topics such as family presence in critical illness [29]. It is nonetheless crucial that future studies explore family presence in an international and multicultural context, as family relationships and the role of family in the healthcare system may vary between countries and cultures, and cultural competence is necessary care that is truly patient-centred [30].

In addition to language limitations, we did not critically appraise the quality of studies, including examination of funding sources that may have biased study results. Presenting numbers for reports that address PPB incidentally is both a strength of this review, in being highly inclusive 
18

16

14

12

10

8

6

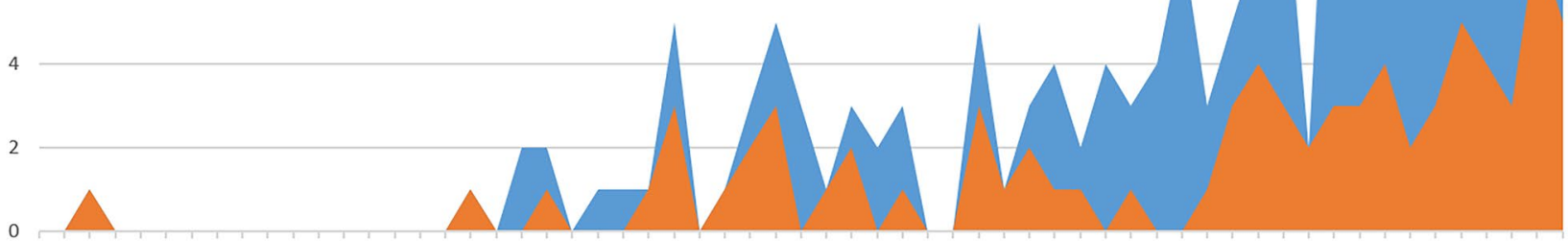

1960196219641966196819701972197419761978198019821984198619881990199219941996199820002002200420062008201020122014201620182020

$\square$ total by year $\square$ direct by year

Fig. 4 Reports by year, total and only those directly assessing an element of PPB (direct)

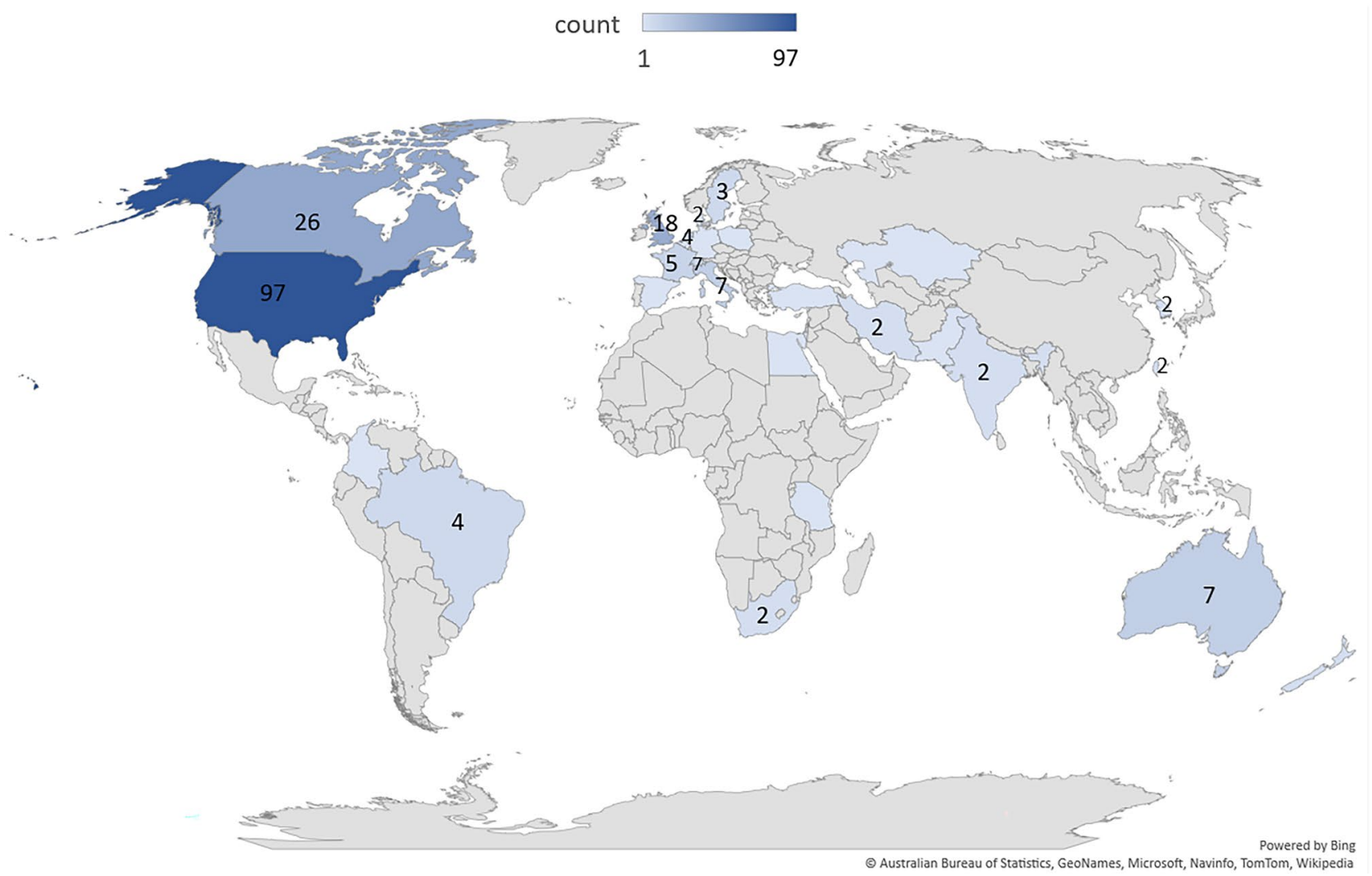

Fig. 5 Reports by country in which work was completed 
and providing future investigators many potential sources to guide future assessment, and a limitation in that it seems likely that we missed some literature, despite performing a highly inclusive full text review. Finally, by focusing on parental presence, rather than that of the entire family, our review is limited in scope. We would advocate for further studies and evidence syntheses that address the entire family of a critically ill child, as their presence or absence may have significant implications for the child's best interest by impacting both the child and their parents [31, 32]

\section{Conclusions}

Although a large body of work addresses PPB of critically ill children, the majority is indirect or as minor findings. Systematic reviews of the literature that directly assesses barriers and enablers to, amount and quality of, and impact and outcomes of parental and family presence at the bedside are warranted to guide best practice and future research.

Supplementary Information The online version contains supplementary material available at https://doi.org/10.1007/s00431-021-04279-6.

Authors' contributions Conceptualization: Jennifer Foster, Jamie Seabrook, Lauren Miller, Kristina Krmpotic, Martha Walls, Corey Slumkoski. Methodology: Jennifer Foster, Lauren Miller, Kristina Krmpotic. Formal analysis and investigation: Lauren Miller, Monique Richard, Annie Kennedy, Jennifer Foster. Writing-original draft preparation: Lauren Miller. Writing-review and editing: Jennifer Foster. Final manuscript critical review: All study team members. Resources and trainee supervision: Jennifer Foster.

Funding LM was supported by the Dalhousie University, Faculty of Medicine Ross Stuart Smith Research in Medicine Summer Studentship, as well as the Dalhousie Medicine Research Foundation Director's Studentship. The funding sources were not involved in any part of the research process.

Data availability Requests for data not supplied in the supplementary material should be directed to the corresponding author.

\section{Declarations}

Consent to participate Not applicable. No participants.

Consent to publication Not applicable. No human subjects.

Ethics approval As an evidence synthesis, this work did not require ethics approval.

Conflicts of interest JF, JS, KK, CS, and MW hold a grant from the Canadian Institutes of Health Research to examine the impact of restricted family presence policies in Canadian pediatric intensive care units during the COVID-19 pandemic, and to provide stakeholder input into optimizing future.

\section{References}

1. Carnevale FA, Gaudreault J (2013) The experience of critically ill children: A phenomenological study of discomfort and comfort. Dynamics 24:19-27

2. UN Commission on Human Rights (46th session) Convention on the Rights of the Child. E/CN.4/RES/1990/74

3. Just AC (2005) Parent participation in care: Bridging the gap in the pediatric ICU. Newborn Infant Nurs Rev 5:179-187. https:// doi.org/10.1053/j.nainr.2005.08.002

4. Franck LS, Callery P (2004) Re-thinking family-centred care across the continuum of children's healthcare. Child Care Health Dev 30:265-277. https://doi.org/10.1111/j.1365-2214. 2004.00412.x

5. Shields L, Pratt J, Hunter J (2006) Family centred care: A review of qualitative studies. J Clin Nurs 15:1317-1323. https://doi.org/ 10.1111/j.1365-2702.2006.01433.x

6. Kaye EC (2021) COVID-19 Caregiver Restrictions in Pediatrics. Hosp Pediatr 11:e12-e14. https://doi.org/10.1542/hpeds. 2020-004291

7. Klompas M (2020) Coronavirus disease 2019 (COVID-19): Protecting hospitals from the invisible. Ann Intern Med 172: 619-620 https://doi.org/10.7326/M20-0751

8. Hill C, Knafl KA, Santacroce SJ (2018) Family-Centered Care From the Perspective of Parents of Children Cared for in a Pediatric Intensive Care Unit: An Integrative Review. J Pediatr Nurs 41:22-33. https://doi.org/10.1016/j.pedn.2017.11.007

9. Smith L, Medves J, Harrison MB, Tranmer J, Waytuck B (2009) The impact of hospital visiting hour policies on pediatric and adult patients and their visitors. JBI Libr Syst Rev 7:38-79. https://doi.org/10.11124/01938924-200907020-00001

10. Sarah B, Mcalvin S, Carew-lyons A (2014) Family prescence during resuscitation and invasive procedures in pediatric critical care: A systematic review. Am J Crit Care 23:477-485. https:// doi.org/10.4037/ajcc2014922

11. Andrist E, Clarke R, Harding M (2020) Paved With Good Intentions: Hospital Visitation Restrictions in the Age of Coronavirus Disease 2019. Pediatr Crit Care Med 21:e924-926. https://doi. org/10.1097/PCC.0000000000002506

12. Litmanovitz I, Silberstein D, Butler S, Vittner D (2021) Care of hospitalized infants and their families during the COVID-19 pandemic: An international survey. J Perinatol 41:981-987. https:// doi.org/10.1038/s41372-021-00960-8

13. Coats H, Bourget E, Starks H et al (2018) Nurses' reflections on benefits and challenges of implementing family centered care in pediatric intensive care units. Am J Crit Care 27:52-58. https:// doi.org/10.4037/ajcc2018353

14. Foster J, AlOthmani F, Seabrook J, AlOfisam T, AlGarni Y, Sarpal A (2018) Parental Presence at the Bedside of Critically Ill Children in a Unit With Unrestricted Visitation. Pediatr Crit Care Med 19:e387-e393. https://doi.org/10.1097/PCC.0000000000001597

15. Smith AB, Hefley GC, Anand KJS (2007) Parent bed spaces in the PICU: Effect on parental stress. Pediatr Nurs 33:215-221

16. Knafl KA, Aamodt AM, Bowers JE, Holaday B (1985) How Families Manage a Pediatric Hospitalization. West J Nurs Res 7:151-176. https://doi.org/10.1177/0092055X8500700202

17. Feudtner C, Walter JK, Faerber JA et al (2015) Good-parent beliefs of parents of seriously ill children. JAMA Pediatr 169:3947. https://doi.org/10.1001/jamapediatrics.2014.2341

18. Fumagalli S, Boncinelli L, Lo Nostro A et al (2007) Reduced cardiocirculatory complications with unrestrictive visiting policy in an intensive care unit: Results from a pilot, randomized trial. Circulation 113:946-952. https://doi.org/10.1161/CIRCULATIONAHA. 105.572537 
19. Rosa RG, Tonietto TF, Da Silva DB et al (2017) Effectiveness and safety of an extended icu visitation model for delirium prevention: A before and after study. Crit Care Med 45:1660-1667. https:// doi.org/10.1097/CCM.0000000000002588

20. Bishop SM, Walker MD, Mark Spivak I (2013) Family presence in the adult burn intensive care unit during dressing changes. Crit Care Nurse 33:14-24. https://doi.org/10.4037/ccn2013116

21. Arksey H, O’Malley L (2005) Scoping studies: Towards a methodological framework. Int J Soc Res Methodol Theory Pract 8:19-32. https://doi.org/10.1080/1364557032000119616

22. Levac D, Colquhoun H, O'Brien KK (2010) Scoping studies: advancing the methodology. Implement Sci 5:69. https://doi.org/ 10.1186/1748-5908-5-69

23. Frankel LR, Hsu BS, Yeh TS et al (2019) Criteria for critical care infants and children: PICU Admission, discharge, and triage practice statement and levels of care guidance. Pediatr Crit Care Med. 20:847-887. https://doi.org/10.1097/PCC. 0000000000001963

24. Evans R, Madsen B (2005) Culture clash: Transitioning from the neonatal intensive care unit to the pediatric intensive care unit. Newborn Infant Nurs Rev. 5:188-193. https://doi.org/10.1053/j. nainr.2005.08.005

25. Michie S, van Stralen MM, West R (2011) The behaviour change wheel: A new method for characterising and designing behaviour change interventions. Implement Sci 6:42. https://doi.org/10.1186/ 1748-5908-6-42

26. Tughan L (1992) Visiting in the PICU: A study of the perceptions of patients, parents, and staff members. Crit Care Nurs Q 15:57-68. https://doi.org/10.1097/00002727-199205000-00007
27. Aronson PL, Yau J, Helfaer MA, Morrison W (2009) Impact of family presence during pediatric intensive care unit rounds on the family and medical team. Pediatrics 124:1119-1125. https://doi. org/10.1542/peds.2009-0369

28. Turner EL, Nielsen KR, Jamal SM, von Saint André-von Arnim A, Musa NL (2016) A review of pediatric critical care in resourcelimited settings: A look at past, present, and future directions. Front Pediatr 4:5. https://doi.org/10.3389/fped.2016.00005

29. Allik J, Lauk K, Realo A (2020) Indicators of the scientific impact of nations revisited. Trames J Humanit Soc 24:231. https://doi.org/ 10.3176/tr.2020.2.07

30. Epner DE, Baile WF (2012) Patient-centered care: The key to cultural competence. In: Annals of Oncology. 23 Suppl 3:33-42. https://doi.org/10.1093/annonc/mds086

31. Terp K, Sjöström-Strand A (2017) Parents' experiences and the effect on the family two years after their child was admitted to a PICU—An interview study. Intensive Crit Care Nurs 43:143-148. https://doi.org/10.1016/j.iccn.2017.06.003

32. Jenkins TL, Landon C, de Almeida ML et al (2006) Impact of Pediatric Critical Illness and Injury on Families: A Systematic Literature Review. Pediatrics. 118:S203-S218. https://doi.org/10. 1542/peds.2006-0951b

Publisher's Note Springer Nature remains neutral with regard to jurisdictional claims in published maps and institutional affiliations.

\section{Authors and Affiliations}

\section{Lauren Miller ${ }^{1} \cdot$ Monique Richard $^{2} \cdot$ Kristina Krmpotic $^{2,3}$ (1) $\cdot$ Anne Kennedy ${ }^{4}$ Jamie Seabrook ${ }^{5,6,7}$ (1) Corey Slumkoski ${ }^{8} \cdot$ Martha Walls $^{8} \cdot$ Jennifer Foster ${ }^{2,3,7}$ (])}

Lauren Miller

lauren.miller@dal.ca

Monique Richard

monique.richard@iwk.nshealth.ca

Kristina Krmpotic

kristina.krmpotic@iwk.nshealth.ca

Anne Kennedy

anniekennedy@live.com

Jamie Seabrook

jseabro2@uwo.ca

Corey Slumkoski

Corey.Slumkoski@msvu.ca

Martha Walls

Martha.walls@msvu.ca

1 Faculty of Medicine, Dalhousie University, Halifax, NS,

Canada
2 Department of Pediatric Critical Care, IWK Health, Halifax, NS, Canada

3 Department of Critical Care, Faculty of Medicine, Dalhousie University, Halifax, NS, Canada

4 School of Education, Acadia University, Wolfville, NS, Canada

5 School of Food and Nutritional Sciences, Brescia University College at Western University, London, ON, Canada

6 Department of Pediatrics and Department of Epidemiology \& Biostatistics, Western University, London, ON, Canada

7 Children's Health Research Institute and Lawson Health Research Institute, London, ON, Canada

8 Department of Pediatric Critical Care Parent Partner, IWK Health, Halifax, NS, Canada 